\title{
Observações sobre aplicações de controle em ecótopo com alta infestação de Culex quinquefasciatus Say (Diptera, Culicidae)
}

\author{
Sirlei Antunes de Morais ${ }^{1}$, Mauro Toledo Marrelli ${ }^{1} \&$ Delsio Natal ${ }^{1}$
}

${ }^{1}$ Departamento de Epidemiologia da Faculdade de Saúde Pública, Universidade de São Paulo, Av. Dr. Arnaldo, 715, 01246-904 São Paulo-SP, Brasil. sirlei@usp.br

\begin{abstract}
Observations on control applications in breeding sites with high infestation of Culex quinquefasciatus Say (Diptera, Culicidae). With the objective of studying aspects of the infestation of the mosquito Culex quinquefasciatus in the Pinheiros river, in the city of São Paulo, SP, Brazil, and to evaluate the effectiveness of the control applications made in the place, activities of collecting mosquitoes were performed in ten sites located along the two margins of the river. The mosquitoes were collected by aspiration method, during the period of one year. After that, they were sent to the laboratory for identification, scoring and treatment of the frequency data comparing with the applications data of chemical and biological control strategies. The control applications had presented partial effect in the population frequency of the species, what opens for a discussion about the peculiarities of the breeding sites, as possible barriers in the efficiency of the products applied in the aquatic environment and in the border vegetation, along the course of the river.
\end{abstract}

KEYWORDS. Control; insecticides; larvae control.

RESUMO. Observações sobre aplicações de controle em ecótopo com alta infestação de Culex quinquefasciatus Say (Diptera, Culicidae). Com o objetivo de conhecer aspectos da infestação do mosquito Culex quinquefasciatus no rio Pinheiros, São Paulo, SP, Brasil, e avaliar a eficácia das aplicações de controle efetuadas no local, foram realizadas atividades de monitoramento da população de mosquitos em dez pontos de amostragens, localizados ao longo das duas margens do rio Pinheiros. Os mosquitos foram coletados por meio de técnica de aspiração à bateria, durante o período de um ano e encaminhados ao laboratório para identificação, contagem e tratamento dos dados de frequiência com as datas de aplicações de controle químico e biológico da população de mosquitos. As aplicações de controle apresentam efeitos parciais na freqüência populacional da espécie, o que abre discussão sobre as peculiaridades do ecótopo, como possíveis barreiras na eficiência dos produtos aplicados no meio aquático e na vegetação marginal, ao longo da calha do rio.

PALAVRAS-CHAVE. Controle; inseticidas; larvicida biológico.

O rio Pinheiros recebe resíduos de inúmeros efluentes domésticos e industriais ao passar pela área metropolitana da cidade de São Paulo. Com isso, os dados de qualidade da água apontam níveis elevados de material mineralizado (SMA 2002), como resultado da progressividade da decomposição da matéria orgânica e industrial, constantemente despejada no seu leito. A água do rio Pinheiros apresenta temperatura elevada, alta concentração de poluentes e praticamente não possui fluxo (SMA 2002). Além disso, emana mau cheiro pela degradação anaeróbia, confirmada pela ausência de oxigênio dissolvido, o que a caracteriza como sendo anóxica.

As características da água e do ambiente às margens do rio Pinheiros propiciam o desenvolvimento e a proliferação da espécie Culex (Culex) quinquefasciatus Say (1823). Esse mosquito tem as formas imaturas adaptadas ao meio aquático poluído e anóxico (Urbinatti et al. 2001), pelo desenvolvimento de mecanismos alternativos de sobrevivência (Clements 1999).

As fêmeas adultas dessa espécie possuem tendência à alimentação com sangue humano (Consoli \& Lourenço-deOliveira 1994), permitindo o seu desenvolvimento em ambiente urbanizado. Além de ser provocador de incômodo para as comunidades que vivem próximos aos seus criadouros (TaipeLagos \& Natal 2003), o mosquito Cx. quinquefasciatus é vetor de vários agentes patogênicos ao homem (Forattini 2002), o que o caracteriza como sendo de elevada importância em Saúde Pública, justificando o seu controle em áreas de infestação.

Durante décadas, o leito e as margens do rio Pinheiros passaram por uma série de aplicações de produtos químicos para o controle da população de mosquitos. A partir da década de 40, foram aplicados inicialmente produtos da classe dos organoclorados e a seguir os organofosforados e piretróides (Bracco et al. 1997). Atualmente, além de outros produtos, são efetuadas aplicações de Bacillus sphaericus White \& Lotay (1980) para o controle larvário de Culex quinquefasciatus.

Neste trabalho são analisados aspectos da infestação da população de mosquitos no rio Pinheiros e avaliada a eficácia das aplicações de controle efetuadas no local, sob responsabilidade do Centro de Controle de Zoonoses, da Prefeitura Municipal de São Paulo (CCZ/PMSP). Ademais, abordam-se sobre as peculiaridades deste ecótopo, como possíveis barreiras na eficiência dos produtos aplicados no meio aquático e na vegetação marginal, ao longo da calha do rio.

\section{MATERIALE MÉTODOS}

Para as atividades de monitoramento e capturas da população de mosquitos foram selecionados dez pontos, 
localizados ao longo das duas margens do rio Pinheiros, na cidade de São Paulo, Estado de São Paulo (Fig. 1). Os pontos foram especificados por ordem numérica. Para a marcação de cada ponto foi instalada uma estaca, sendo que a região de borda explorada correspondeu um raio de 50m desta marcação.

Para as capturas, utilizou-se aspirador à bateria do tipo adaptado por Nasci (1981), passado lentamente em meio à vegetação de borda, por um período de cinco minutos. Após cada captura, os mosquitos foram mortos imediatamente e colocados em caixas entomológicas de plástico, embaladas em isopor com gelo reciclável. No laboratório, os mosquitos foram contados e identificados, com base nas chaves taxonômicas de Forattini (2002).

As capturas de mosquitos adultos foram efetuadas semanalmente, durante doze meses, de agosto de 2003 a julho de 2004, nos pontos 4, 8 e 10. Nos pontos 3, 7 e 9, as capturas foram realizadas durante dez meses, no período de outubro de 2003 a julho de 2004. Nos demais pontos (1,2, 5 e 6), as capturas foram realizadas de outubro de 2003 a março de 2004.

Os deslocamentos até os locais de coleta e o planejamento do período de amostragem foram efetuadas pelo CCZ/PMSP, obedecendo-se períodos intermitentes em cada ponto, com coletas semanais e também a disponibilidade do setor.

Os dados de frequiência obtidos neste estudo foram utilizados para comparação em justaposição com os produtos inseticidas aplicados constantemente em toda a extensão do leito do rio e na área que o circunda. Segundo o CCZ/PMSP é aplicado no local inseticidas da classe dos organofosforados, como o larvicida à base de Temefós, o Abate ${ }^{\circledR}$ (aleatoriamente, em alguns pontos) e adulticidas piretróides, como a Cipermetrina (aleatoriamente, em alguns pontos) e Betacyfluthrin (diariamente, em toda a margem do rio) e também o larvicida biológico à base de Bacillus sphaericus, Vectolex (B) $\mathrm{G}$ (mensalmente, em toda a extensão do rio). Os produtos foram aplicados em conformidade às orientações dos fabricantes.

Segundo o CCZ/PMSP, a escolha da formulação granulada de $B$. sphaericus foi devida principalmente à alta tecnologia de encapsulamento e menor densidade das partículas do produto, que permite a este permanecer mais tempo na superfície líquida, no limiar de alimentação das larvas do $C x$. quinquefasciatus. E ainda, a exemplo de outras experiências, como a do Recife (Regis et al. 1995), optou-se pelo larvicida como complemento, após inúmeras tentativas de controle com produtos químicos.

Para a análise estatística foram utilizados os programas: SPSS ${ }^{\circledR}$ 12.0 for Windows e SAS® 9.1.3 for Windows. Com os dados de freqüência dos mosquitos adultos foram efetuados cálculos de estatística descritiva (médias, desvio padrão, mínimo, máximo), teste de hipóteses, análises de variância para comparação entre os pontos de coleta e teste de comparação de médias (Teste de Tukey).

\section{RESULTADOS EDISCUSSÃO}

Distribuição de Cx. quinquefasciatus no rio Pinheiros.
Durante o período de estudo foram capturados 104.318 mosquitos adultos nos dez pontos localizados ao longo das duas margens do rio Pinheiros (Fig. 1). Os resultados da identificação dos mosquitos adultos mostraram que a espécie $C x$. quinquefasciatus apresentou exclusividade entre os culicídeos.

O ponto 2, localizado no extremo norte do rio, apresentou frequiência maior de mosquitos adultos, com um total de 19.717 mosquitos capturados e máximo de 541 mosquitos por minuto de aspiração. $O$ ponto que apresentou menor frequiência foi o ponto 10 , no extremo sul do rio, com um total de 257 mosquitos capturados e máximo de 13 mosquitos por minuto.

A distribuição no ponto 2 foi mais dispersa comparada com os outros pontos de captura. As médias da freqüência de mosquitos capturados são significativamente diferentes para o ponto 2 com relação aos outros pontos $(\mathrm{p}<0,05)$ (comparação de médias de Tukey). Somente não houve diferenças entre o ponto 2 e o ponto $4(p>0,05)$. Não houve diferenças significativas entre os outros pontos de captura $(p>0,05)$.

Estudos da distribuição dos mosquitos no rio Pinheiros apontam que as diferenças na freqüência podem estar relacionadas à tendência da água a levar poluentes e larvas para a parte norte do canal, após períodos de chuva. Haja vista que em épocas de estiagem ocorrem picos de freqüência semelhantes em toda a extensão do rio. Além disso, são ressaltadas as diferenças na qualidade da água entre as extremidades do canal e as implicações na proliferação de mosquitos (Morais et al. 2006).

Efeitos das aplicações dos produtos inseticidas na população de mosquitos. A Fig. 2 mostra a flutuação da população de mosquitos adultos de $C x$. quinquefasciatus às margens do rio Pinheiros, em justaposição com as aplicações dos produtos inseticidas. Observa-se que a população de adultos de $C x$. quinquefasciatus às margens do rio Pinheiros possui flutuação sazonal, com intervalos na curva populacional entre as diferentes gerações, como resultado do envelhecimento dos mosquitos, dos períodos mais frios e chuvosos do ano (Clark et al. 1967) ou devido ao efeito produzido pelas aplicações de inseticidas. Ademais, possui eventos de explosão, seguida de dispersão em massa dos mosquitos.

Durante o período de estudo, foram efetuadas quatro aplicações do larvicida organofosforado Temefós (Pontos 1, 3 e 4) (Fig. 2). Nos pontos 1 e 3 as aplicações se deram anteriormente à ocorrência de um intervalo na curva populacional, ocorrido entre os meses de outubro e novembro. Ainda no ponto 3, a aplicação feita no início de maio, foi efetuada posteriormente a um pico de explosão da população de mosquitos, ocorrido também em outros pontos do rio. Essa explosão ocorreu durante uma queda na pluviosidade, após um período de chuvas (Tab. I). Ocasionalmente, nos pontos 7 , 8 e 9 , a freqüência de mosquitos adultos apresentou o mesmo comportamento, entretanto, sem a aplicação do larvicida.

Pesquisas apontam (Clements 1999) que as larvas de $C x$. quinquefasciatus tendem a desenvolver mecanismos 
alternativos no modo de alimentação, podendo ser altamente seletivas às partículas presentes no meio líquido. Pelo exposto, esta linhagem pode ter um mecanismo de alimentação seletivo, adicionado ao fato de que a água do rio Pinheiros, enquanto altamente poluída e sem fluxo, propicia a rápida precipitação do inseticida antes que este venha a ser ingerido pelas larvas.

As aplicações do adulticida piretróide Betacyfluthrin, efetuadas diariamente nas margens do rio, também apresentou pouca influência na população de mosquitos, haja vista a análise dos valores na curva populacional, onde a frequiência de mosquitos adultos se manteve no padrão esperado, em uma série temporal.

Nas aplicações de adulticidas na vegetação marginal do rio, deve-se levar em consideração o grande potencial do meio aquático como criadouro de imaturos. E também o fato de que as aplicações deste produto não atingem parte da população de fêmeas ingurgitadas e com ovos, uma vez que estas tendem a permanecer nos locais de alimentação de sangue, nas áreas residenciais (Forattini 2002).

Durante o período de estudo, foram efetuadas algumas aplicações de $B$. sphaericus, seguidas de picos explosivos na população de mosquitos (coletas 11 e 14, ponto 2) (Fig. 2). Nesses casos, e também em outras ocasiões, as aplicações do produto não contiveram picos característicos de época de verão.

Em determinados pontos de coleta tanto as aplicações de B. sphaericus, como as de Cipermetrina foram efetuadas após a ocorrência de picos explosivos, seguidas de redução brusca na freqüência de adultos (coletas 13 e 18, ponto 2) (Fig. 2). Estes mosquitos tendem a dispersar-se em virtude de sua própria dinâmica, na mesma rapidez com que se desenvolveram. É conhecido que voam em massa, em direção a abrigos mais favoráveis, em outros pontos, ao longo da extensão do rio. Essa condição pode estender-se para a questão da disponibilidade de abrigos e o manejo destes na área da margem do rio.

Nos períodos entre os meses de outubro e novembro, março e abril e junho e julho as aplicações tanto de B. sphaericus, como de Cipermetrina foram seguidas de queda contínua na freqüência de mosquitos adultos (Fig. 2). Essas reduções nos valores de freqüência podem ter ocorrido em parte pelas aplicações dos produtos inseticidas, em parte pelo envelhecimento natural da população de mosquitos (outubro e novembro) e também pelos níveis elevados de chuva (março e abril), que interferem diretamente na freqüência da espécie (Clark et al. 1967). No período entre os meses de junho e julho as baixas temperaturas da época de inverno também são condições fortemente contribuintes para reduzir a população de mosquitos.

No mês de agosto, no ponto de coleta número 10 (Fig. 2), houve uma explosão de mosquitos e, no restante do ano, uma redução acentuada e de forma contínua na freqüência da população. Durante esse período foram efetuadas nove aplicações de B. sphaericus. Entretanto, apesar da água do rio, neste ponto, se adequar ao desenvolvimento de $C x$. quinquefasciatus, foram efetuadas limpezas contínuas na

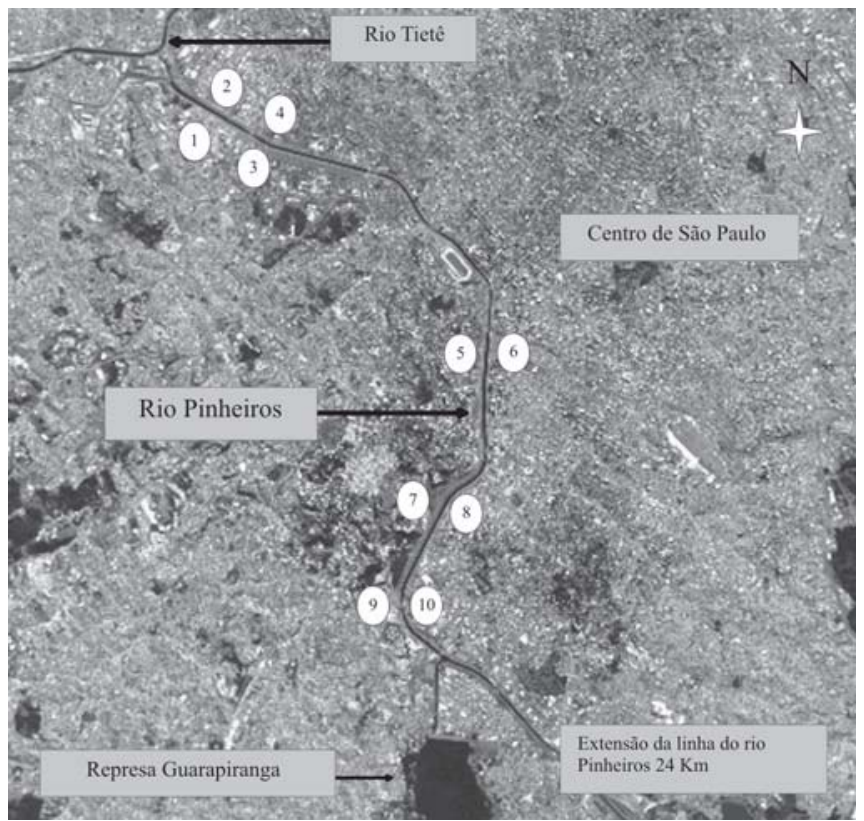

Fig. 1. Extensão do rio Pinheiros, São Paulo, com indicação dos pontos de amostragem para coleta de adultos de CX. quinquefasciatus. Imagem do satélite SPOT, 2002, cedida pela Intersat S/A.

vegetação, às margens do rio (CCZ/PMSP), o que interferiu na contagem das amostras de mosquitos adultos.

O ponto de coleta número 9 (Fig. 2) apresentou grandes intervalos na frequiência de mosquitos adultos entre os meses de fevereiro e abril, sendo que foi efetuada uma aplicação de B. sphaericus, anterior a este período. Nessa mesma ocasião ocorreram os maiores picos de pluviosidade (Tab. I), o que pode ter carreado as formas imaturas dos mosquitos a partir deste ponto para a outra extremidade do rio. Além disso, essa queda na frequiência de mosquitos não pode ser interpretada somente como efeito do produto, uma vez que, com muitas chuvas, este é diluído na água, reduzindo ainda mais sua efetividade.

Eficiência de B. sphaericus nas águas do rio Pinheiros. Alguns estudos apontam resultados positivos na utilização do entomopatógeno à base de B. sphaericus para o controle de Cx. quinquefasciatus (Regis et al. 1995; Lacey \& Singer 1982). Entretanto, os ecótopos explorados possuem características diferentes das encontradas no rio Pinheiros, na cidade de São Paulo.

Segundo Mulla et al. (1984), a eficácia dos produtos com agentes biológicos depende de fatores ambientais, tais como a qualidade da água do criadouro, a riqueza e os tipos de nutrientes do meio líquido, o clima, a densidade larvária, entre outros.

A temperatura é um dos fatores relevantes na eficiência do B. sphaericus. Decréscimos substanciais na temperatura são usualmente relatados para uma redução na atividade larvicida. Carvalho Pinto \& Consoli, citado por Consoli et al. (1995), testaram cepas de Bs 2362 para $C x$. quinquefasciatus em 

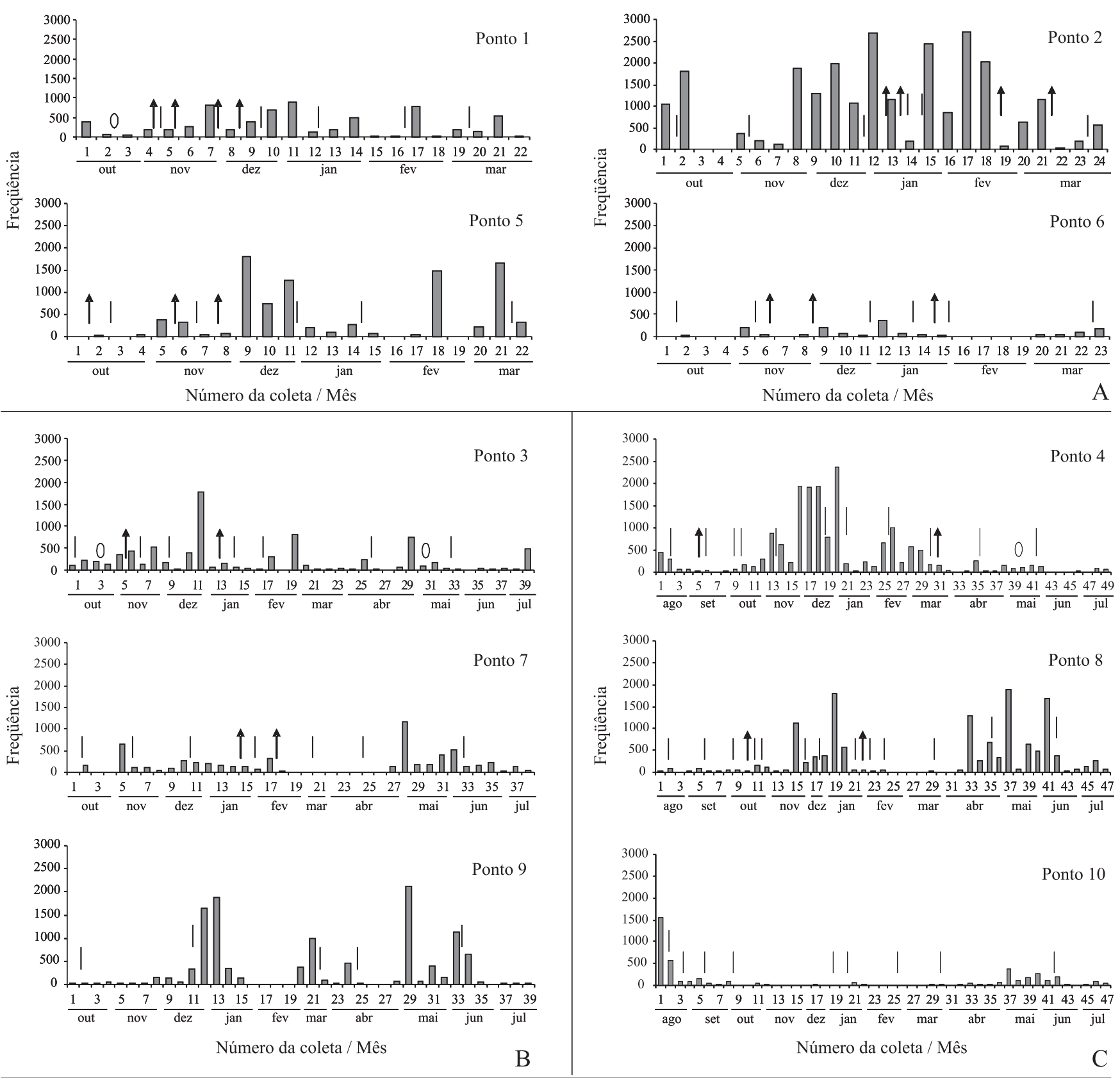

$\uparrow$ Cipermetrina 0 Temefós | Bacillus sphaericus

Fig. 2. Distribuição das frequiências de adultos de $C x$. quinquefasciatus no rio Pinheiros e aplicação de inseticida, por ponto de coleta, segundo mês e número da coleta. A: pontos com coletas efetuadas no período de outubro de 2003 a março de 2004. B: outubro de 2003 a agosto de 2004. C: agosto de 2003 a julho de 2004 .

temperaturas de 15,20 e $25^{\circ} \mathrm{C}$ e observaram uma redução de LC50 com o aumento da temperatura. As águas do rio Pinheiros apresentam variações na temperatura entre 17 a $30^{\circ} \mathrm{C}$, com picos de até $35^{\circ} \mathrm{C}$ no verão (SMA 2002). Os picos mais altos de temperatura estão na extremidade sul da calha do rio. Essas condições devem ser consideradas no planejamento das aplicações do entomopatógeno, uma vez que aceleram a degradação deste, reduzindo sua atividade larvicida.

Em estudos preliminares realizados dentro de uma zona intertropical, onde foi aplicada a cepa de B. sphaericus 2362 contra larvas de $C x$. quinquefasciatus em coleções de água, próximo ao meio urbano, na Vila Iaundé, sul de Camarões, África; Hougard et al. (1993) constataram que nos períodos de maior densidade de mosquitos o impacto da aplicação teve maior efeito.

Somando-se a isso, para Hougard et al. (1993), o padrão de chuvas, as condições em que o produto foi aplicado e a reinvasão de mosquitos de áreas não tratadas foram os principais fatores limitantes da eficácia do produto. A estratégia adotada depende das particularidades sócio-geográficas e de um estudo 
Tabela I. Distribuição mensal das temperaturas máxima e mínima, e Coeficiente de Variação e Pluviosidade, no rio Pinheiros, SP. Agosto de 2003 a julho de 2004.

\begin{tabular}{cccc}
\hline \multirow{2}{*}{ Mês } & Temp. Máx. & Temp. Mín. & Pluviosidade \\
\cline { 2 - 4 } & ${ }^{\circ} \mathrm{C}(\mathrm{CV})$ & ${ }^{\circ} \mathrm{C}(\mathrm{CV})$ & $\mathrm{mm}$ \\
\hline ago & $19,9(26,9)$ & $11,3(22,8)$ & 0,7 \\
set & $22,5(28,8)$ & $13,8(18,4)$ & 0,9 \\
out & $24,5(20,0)$ & $15,5(15,5)$ & 2,3 \\
nov & $25,5(18,4)$ & $16,8(15,7)$ & 6,5 \\
dez & $27,4(17,9)$ & $18,3(9,3)$ & 5,8 \\
jan & $24,9(14,3)$ & $18,0(9,1)$ & 9,8 \\
fev & $25,5(15,2)$ & $18,0(8,5)$ & 12,5 \\
mar & $25,3(12,3)$ & $17,8(11,4)$ & 7,5 \\
abr & $25,6(11,5)$ & $18,2(11,0)$ & 13,8 \\
mai & $20,7(16,9)$ & $13,8(20,7)$ & 6,9 \\
jun & $21,3(17,5)$ & $12,3(19,4)$ & 9,5 \\
jul & $19,6(23,3)$ & $12,2(22,1)$ & 9,2 \\
\hline
\end{tabular}

Fonte: EMAE/SP

aprofundado da dinâmica da população de mosquitos.

No caso do rio Pinheiros, além do comentado, outros fatores devem ser considerados na eficiência do larvicida, como por exemplo: a grande disponibilidade de substâncias com íons livres e, portanto, reagentes. As águas do rio Pinheiros apresentam níveis elevados de condutividade elétrica, sendo estes ainda maiores na parte norte da calha do rio (até 560uS) (SMA 2002). Com isso, o princípio ativo de $B$. sphaericus, sendo biológico, tende a degradar-se ainda mais rapidamente sob meio líquido com alto potencial químico (Consoli et al. 1995).

Outros fatores podem ser considerados, como a grande concentração de poluentes no meio, aderência do produto às partículas do sedimento e a pouca movimentação da água, que propicia maior rapidez na sedimentação das substâncias larvicidas.

Para maior efetividade do larvicida biológico, sugere-se a elaboração de um modelo de protocolo, com provisão de testes de campo, segundo os protocolos padrão da WHO (1982), tendo como base a ecologia e biologia da população de mosquitos. Este modelo pode servir de referência no conhecimento da regulação natural da população, de modo a identificar o impacto causado pelas aplicações de controle.

Para tanto devem ser testadas e observadas as características gerais da água, assim como a realização de ensaios de aderência do produto às partículas do sedimento, natureza do sedimento, tempo de precipitação e toxicidade do produto no meio líquido.

Ressalta-se a importância de análises do conteúdo do estômago da larva, a fim de verificar se a população de imaturos de $C x$. quinquefasciatus está incluindo os esporos na sua alimentação diária.

As técnicas utilizadas para o controle de $C x$. quinquefasciatus são geralmente adotadas com base nos programas já existentes para o combate de Aedes aegypti (Linnaeus) e Aedes albopictus (Skuse). Em vista disso, devemse levar em consideração os ecótopos e o comportamento de Cx. quinquefasciatus, pois estes ocupam criadouros típicos, suas larvas possuem um modo diferente de alimentação e as fêmeas adultas desenvolveram, ao longo do tempo, hábitos diferentes na alimentação de sangue (Clements 1999). Para o planejamento de ações de controle da população, sugere-se a utilização e o estudo de novas técnicas, específicas para $C x$. quinquefasciatus.

Conclusões. Os resultados apontam alta infestação de $C x$. quinquefasciatus no rio Pinheiros, com desenvolvimento da população de forma explosiva. A potencialidade das águas do rio Pinheiros para o desenvolvimento desses mosquitos ultrapassam os resultados produzidos pelas aplicações de controle na população.

As aplicações de produtos adulticidas e larvicidas nas margens e na água do rio, de um modo geral, apresentam efeitos parciais na frequiência dos mosquitos adultos. Esse fato é confirmado pelas características da curva populacional de $C x$. quinquefasciatus e pela ocorrência de séries explosivas dos mosquitos, após a aplicação dos produtos.

Os principais fatores limitantes para a efetividade dos produtos inseticidas podem estar relacionados ao padrão de chuvas, à concentração e os meios em que estes são aplicados, a reinvasão de mosquitos de áreas não tratadas, às particularidades da água do rio, como os níveis de condutividade elétrica e temperatura; e também, a falta de conhecimento da suscetibilidade dos mosquitos aos produtos e às tendências do modo de alimentação das larvas de Culex quinquefasciatus.

Agradecimentos. À equipe do Centro de Controle de Zoonoses (CCZ) da Prefeitura Municipal da Saúde, pela colaboração nos trabalhos de campo e às informações cedidas.

\section{REFERÊNCIAS}

Bracco J. E.; M. Dalbon; O. Marinotti \& J. M. S. Barata. 1997. Resistência a inseticidas organofosforados e carbamatos em população de Culex quinquefasciatus. Revista de Saúde Pública 31: 182-3.

Clark L. R.; P. W. Geier; R. D. Hugles \& R. F. Morris. 1967. Ecology of insect populations in theory and practice. London, Methuen, $232 \mathrm{p}$.

Clements A. N. 1999. The biology of mosquitoes: sensory reception and behaviour. Wallingford, CABI, v. 2., $740 \mathrm{p}$.

Consoli R. A. G. B.; C. J. Carvalho-Pinto; M. A. Oliveira; B. S. Santos; M. A. Lamounier; R. S. A. Alves; C. M. B. Silva \& L. Rabinovitch 1995. Some environmental and biological factors influencing the activity of entomopathogenic Bacillus on mosquito larvae in Brazil. Memórias do Instituto Oswaldo Cruz 90: 121-24.

Consoli R. A. G. B. \& R. Lourenço-de-Oliveira. 1994. Principais mosquitos de importância sanitária no Brasil. Rio de Janeiro, Fiocruz, 225 p.

Forattini OP. 2002. Culicidologia Médica: identificação, biologia e epidemiologia. São Paulo, EDUSP, v. 2., 860 p.

Hougard J. M.; R. Mbentengam; L. Lochouarn; H. Escaffre; F. Darriet; P. Barbazan \& D. Quillévéré. 1993. Lutte contre Culex quinquefasciatus par Bacillus sphaericus: resultants d'une campagne pilote dans une grande agglomeration urbaine d'Afrique équatoriale. WHO Bulletin 71: 357-75

Lacey L. A. \& S. Singer. 1982. Larvicidal activity of new isolates of Bacillus sphaericus and Bacillus thurringiensis $(\mathrm{H}-14)$ against 
anopheline and culicine mosquitoes. Mosquito News 42: 537-543.

Morais S. A.; M. T. Marrelli \& D. Natal. 2006. Aspectos da distribuição de Culex (Culex) quinquefasciatus Say (Diptera, Culicidae) na região do rio Pinheiros, na cidade de São Paulo, Estado de São Paulo, Brasil. Revista Brasileira de Entomologia 50: 413-18.

Mulla M. S.; H. A. Darwazeh; E. W. Davidson; H. T. Dulmage \& S. Singer. 1984. Larvicidal activity and field efficacy of Bacillus sphaericus strains against mosquito larvae and safety to nontarget organisms. Mosquito News 44: 336-42.

Nasci R. S. 1981. A light weight battery powered aspirator for collecting resting mosquitoes in the field. Mosquito News 41: 808-11.

Regis, L.; M. H. N. L. Silva-Filha; C. M. F. Oliveira; E. M. Rios; S. B. Silva \& A. Furtado. 1995. Integrated control measures against Culex quinquefasciatus, the vector of filariasis in Recife. Memórias do Instituto Oswaldo Cruz 90: 115-19.
[SMA] São Paulo. Secretaria do Estado do Meio Ambiente. Coordenadoria de Planejamento Ambiental. 2002. Informações básicas para o planejamento ambiental. São Paulo, SMA, 84p.

Taipe-Lagos, C.B.; D. Natal. 2003. Abundância de culicídeos em área metropolitana preservada e suas implicações epidemiológicas. Revista de Saúde Pública 37: 275-9.

Urbinatti, P. R.; S. Sendacz \& D. Natal. 2001. Imaturos de mosquitos (Díptera: Culicidae): caracterização de criadouros - Parque Ecológico do Tietê, Guarulhos, SP. Revista de Saúde Pública 35: 461-6.

[WHO] World Health Organization. 1982. The role of biological agents in integrated vector control and the formulation of protocols for field testing of biological agents. Report of the sixth meeting of the scientific working group on biological control of vectors. WHO/TDR/VEC-SWG (6)/82.3, $46 \mathrm{pp}$. 